\title{
Time-Multiplexing CNN Simulator
}

\author{
Chi-Chien Lee and José Pineda de Gyvez \\ Department of Electrical Engineering, \\ Texas A\&M University,College Station, Texas 77843-3128 USA
}

\begin{abstract}
A novel approach to simulate Cellular Neural Networks (CNN) is presented in this paper. The approach, time-multiplexing simulation, is prompted by the need to simulate hardware models and test hardware implementations of CNN. For practical size applications, due to hardware limitations, it is impossible to have a one-on-one mapping between the $\mathrm{CNN}$ hardware processors and all the pixels of the image. This simulator provides a solution by processing the input image block by block, with the number of pixels in a block being the same as the number of CNN processors in the hardware. The algorithm for implementing this simulator is also presented, along with some simulation results and comparisons.
\end{abstract}

\section{INTRODUCTION}

The basic circuit unit of $\mathrm{CNN}$ is called a cell [1]. It contains linear and nonlinear circuit elements. Any cell, $C(i, j)$, is connected only to its neighbor cells, i.e. adjacent cells interact directly with each other. This intuitive concept is called neighborhood and is denoted as $N(i, j)$. Cells not in the immediate neighborhood have indirect effect because of the propagation effects of the dynamics of the network. Each cell has a state $x$, input $u$, and output $y$. The state of each cell is bounded for all time $t>0$ and, after the transient has settled down, a cellular neural network always approaches one of its stable equilibrium points. This last fact is relevant because it implies that the circuit will not oscillate. The dynamics of a CNN has both output feedback $(A)$ and input control $(B)$ mechanisms. The first order nonlinear differential equation defining the dynamics of a cellular neural network cell can be written as follows

$C \frac{d x_{i j}(t)}{d t}=-\frac{1}{R} x_{i j}(t)+\sum_{C(k, l) \in N\left(i_{j}\right)} A(i, j ; k, l) y_{k j}(t)+\sum_{C(k, l) \in N\left(i_{i j}\right)} B(i, j ; k, l) u_{k l}$

$y_{i j}(t)=\frac{1}{2}\left(\left|x_{i j}(t)+1\right|-\left|x_{i j}(t)-1\right|\right)$

where $x_{i j}$ is the state of cell $C(i, j), x_{i j}(O)$ is the initial condition of the cell, $C$ is a linear capacitor, $R$ is a linear resistor, $I$ is an independent current source, $A(i, j ; k, l) y_{k l}$ and $B(i, j ; k, l) u_{k l}$ are voltage controlled current sources for all cells $C(k, l)$ in the neighborhood $N(i, j)$ of cell $C(i, j)$, and $y_{i j}$ represents the output equation.

For software simulation purposes, equation (1) is solved within each cell, in a discretized form, to simulate its state dynamics. One common way of processing a large complex image is using a raster approach [2]. This approach implies that each pixel of the image is mapped onto $\mathrm{a} C N \mathrm{~N}$ processor. That is, we have an image processing function in the spatial domain that can be expressed as:

$g(x, y)=T(f(x, y))$

where $f(\cdot)$ is the input image, $g(\cdot)$ the processed image, and $T$ is an operator on $f(\cdot)$ defined over the neighborhood of $(x, y)$. From the hardware implementation's point of view, this is a very exhaustive approach. For practical applications, in the order of 250,000 pixels, the hardware would require an enormous amount of processors which would make its implementation unfeasible. An alternative to this scenario is to multiplex the image processing operator.

\section{TIME-MULTIPLEXING SIMULATION}

Under this approach one can define a block of $\mathrm{CNN}$ processors which will process a subimage whose number of pixels is equal to the number of CNN processors in the block. The processing within this subimage follows the raster approach described in the companion paper [2]. Once convergence is achieved, a new subimage is processed. This procedure is repeated until the whole image has been scanned. It is obvious that with this approach the hardware implementation becomes feasible since now the number of CNN processors is finite. Also, the entire image is scanned only once since each block is allowed to fully converge.

Even though the approach seems tempting, an important observation is necessary: The processed border pixels in each subimage may have incorrect values since they are processed without neighboring information. Fortunately, the latency of $\mathrm{CNNs}$ is such that only local interactions are important. Hence, to cope with the previous problem, two sufficient conditions must be considered while doing time-multiplexing simulation. In other words, to ensure that 
each border cell properly interacts with its neighbors it is necessary: 1) to have a belt of pixels from the original image around the subimage, and 2) to have pixel overlaps between adjacent subimages.

It is possible to quantize the processing error of any border cell $C_{i j}$ with neighborhood radius of 1 . Let us compute independently the error due to the feedforward operator and then due to interactions among cells for two horizontally adjacent processing blocks. The absolute processing error due only to the effect of the $B$ template is obtained by subtracting the erroneous state value from the error free states using eq 1 . This yields,

$\varepsilon_{i j}^{B}=\sum_{i=1}^{i=3} b_{i, j+1} \operatorname{sign}\left(u_{i, j+1}\right)$

where $b_{i, j+1}$ are the missing entries from the B template due to the absence of input signals $u_{i, j+1}$ and $\operatorname{sign}(\cdot)$ is the sign function. The latter function is used to represent the status of a pixel, e.g. black $\equiv 1$ and white $\equiv-1$. Notice that the error is both image and template dependent. In other words, the steady state of a border cell may converge to an incorrect value due to the absence of it's neighbors weighted input. Given the local interconnectivity properties of CNN, one can conclude that the minimum width of the input belt of pixels is equal to the neighborhood radius of the CNN.

Let us study now the interactions among cells. For this effect, we can compute the absolute error in a similar form. Disregarding for the moment the $\mathrm{B}$ template this error is

$\varepsilon_{i j}^{A}=\sum_{i=1}^{i=3} a_{i, j+1} y_{i, j+1}(t)$

where $a_{i, j+1}$ are the missing entries from the A template due to the absence of weighted output signals $y_{i, j+l}(\mathrm{t})$. The problem in this situation is more involved because the output signals depend on the state of their corresponding cells. To minimize the error an overlap of pixels between two adjacent blocks is proposed. The minimum overlap width must be proportional to $2 \times$ the neighborhood's radius of the $\mathrm{CNN}$.

The general time-multiplexing procedure consists in iterating each block (subimage) until all CNN cells within the block converge. The block with converged cells will have state variables $x$ which are the values used for the final output image. In the overlapping procedure the left side of the overlapped cells take converged values from Block $_{i}$ and the right side from $B l o c k_{i+1}$, see Fig. 1. Furthermore, the initial conditions for the border cells of $B l o c k_{i+1}$ are the state values obtained while processing $B l o c k_{i-1}$. In our simulator the number of overlapping columns or rows between the adjacent blocks is defined by the user. Even though higher number of overlapping columns or rows means more accurate simulation of neighboring effects on the border cells, for applications where the correct final state is of more importance than the transient states, an overlap of two is usually sufficient. An even number overlapping of overlapping cells is recommended, since the converged cells in the overlapped region can be evenly divided by the two adjacent blocks.

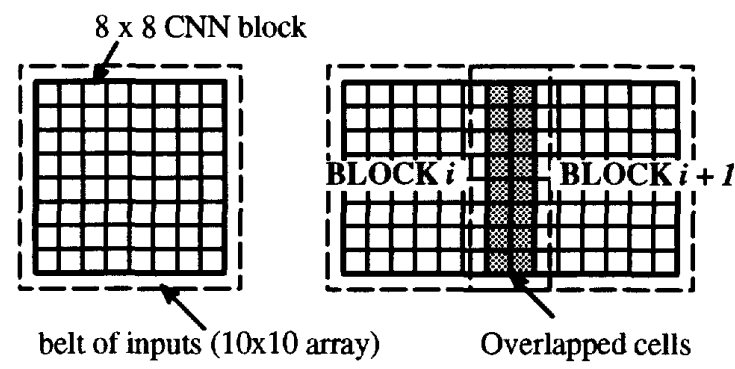

Fig. 1. CNN multiplexing with overlapped cells

With the added overlapping feature, better neighboring interactions are achieved, but at the same time, an increase in computation time is inevitable. However, by taking advantage of the fact that the original input image is been divided into small $\mathrm{CNN}$ subimages, the chance of a subimage having all its pixels black or white is high. This is another feature that can be added to the time-multiplexing simulation to improve computation times. The savings in simulation time come from avoiding repetitive simulations of all-black and all-white subimages.

The idea behind this time-saving scheme is that when the very first all-black/all-white block is encountered, after processing that block, the final states of the block are stored separately from the whole image. When subsequent all-black/all-white blocks are found, there is no need to simulate these blocks since the converged states are readily available in memory, thus avoiding the most time consuming part of the simulation which is the numerical integration.

For the purpose of better understanding the overall idea of this simulation approach, the simplified algorithm is presented below: 
Algorithm: (Time-Multiplexing CNN simulation)

$\mathscr{B}=\left\{C_{i j} \mid i=1, \ldots\right.$, block_x $\wedge j=1, \ldots$, block_y $\}$

$\mathscr{P} \subset \mathscr{B}=$ set of border cells (lower left corner)

overlap = number of cell overlaps;

belt $=$ width of input belt

$M=$ number of rows of the image

$N=$ number of columns of the image

for $(\mathrm{i}=0 ; \mathrm{i}<\mathrm{M} ; \mathrm{i}+=$ block_x - overlap)

for $(j=0 ; j<N ; j+=$ block_y - overlap)

f

/* load initial conditions for the cells in the block except for those in the borders */

for ( $\mathrm{p}=-$ belt; $\mathrm{p}<$ block_ $\mathrm{x}+$ belt; $\mathrm{p}++)$

for $(q=-$ belt; $q<$ block_y + belt; $q++)\{$

$x_{i+p j+j}\left(t_{n}\right)=\left\{\begin{array}{r}u_{i j} \\ 1 \\ -1\end{array} \forall C_{i+p j+j} \in \mathscr{B}\right.$

\} $/$ end for */

${ }^{*}$ if the block is all white or black don't process it */

if $\left(\mathrm{x}_{\mathrm{i}+\mathrm{p}, \mathrm{j}+\mathrm{q}}=-1 \vee \mathrm{x}_{\mathrm{i}+\mathrm{p}, \mathrm{j}+\mathrm{q}}=1 \forall C_{i+p,+q} \in \mathscr{B}\right)$

\{

obtain the final states from memory; continue; )

do $\{/ *$ normal raster simulation $* /$

for $(\mathrm{p}=0 ; \mathrm{p}<$ block_x; $\mathrm{p}++)\{$

for $(q=0 ; q<$ block_y; $q++)$

$\left\{{ }^{*}\right.$ calculation of the next state excluding the belt of inputs */

$x_{i+p j+q}\left(t_{n+1}\right)=x_{i+p j+q}\left(t_{n}\right)+$

$$
+\int_{i_{n}}^{t_{n+1}} f^{\prime}\left(x_{i+p j+q}\left(t_{n}\right)\right) d t
$$

$\forall C_{i+p,+q} \in \mathscr{B}$

* convergence criteria $* /$

if $\left(\frac{d x_{i+p j+q}\left(t_{n}\right)}{d t}=0\right.$ and $y_{k l}= \pm 1$

$\left.\forall C(k, l) \in N_{r}(i+p, j+q)\right)($ converged_cells++;

\} / end for */

${ }^{*}$ update state values $* /$

$x_{i+p j+q}\left(t_{n}\right)=x_{i+p j+q}\left(t_{n+1}\right) \forall C_{i+p j+q} \in \mathscr{B}$

I

J while( converged_cells < (block_x *block_y));

$/ *$ store new state values excluding the ones

corresponding to the border cells $* 1$

$$
\begin{aligned}
& \mathcal{A} \leftarrow x_{i j} \forall C_{i j} \in \mathscr{B} \backslash \mathscr{P} \\
& \text { / } / \text { end for } * /
\end{aligned}
$$

\section{SIMULATION RESULTS AND COMPARISONS}

The general features of the raster CNN simulator [2] are preserved in the time-multiplexing simulator, namely the choice of three integration methods, the format of the input image file and the capability of processing any size of input image. Some representative simulation results due to the effects of the added features in the time-multiplexing simulator are presented in this section.

In the time-multiplexing simulation involving the time-saving scheme, the number of all-black/all-white blocks that will be encountered during a simulation depends on the image itself and the block size chosen by the user. For example, Fig. 2a evidently will benefit from this time-saving scheme, especially from not needing to simulate more than once the all-white block.

Using actual numbers can easily show how much improvement is achieved. The size of Fig. $2 a$ is $395 \times 403$ $(159,185$ pixels), and an edge detection template is used for simulation comparisons. First, using the raster simulator presented in [2], the simulation took 243.51 secs. Next, with the regular time-multiplexing simulator (with overlapping and input belt) the simulation took 363.28 secs. Finally, the time-multiplexing with the time-saving scheme performed the same simulation in 244.22 secs, almost a $33 \%$ improvement from the regular time-multiplexing. The size of the block used was $10 \times 10$, with two rows/columns overlapping.

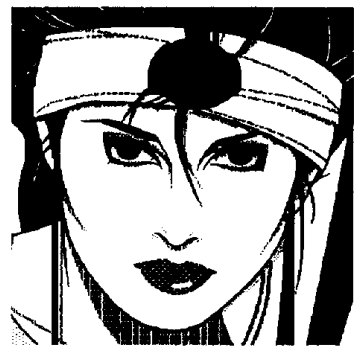

(a)

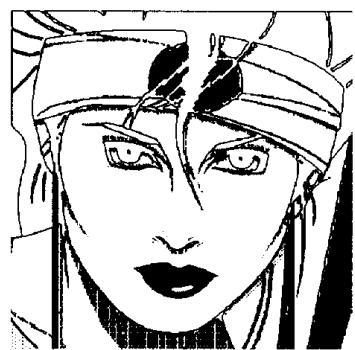

(b)
Fig. 2. Time-multiplexing CNN simulation.
(a) Original image.
(b) After Edge Detection

By taking the lower right-hand corner of Fig. 2a, the cropped image in Fig. 3a, we can easily visualize the effects that the overlapping pixels and the belt of inputs have on the simulation. By choosing the block size to be $10 \times 10$, and 
applying an edge detection template, the results obtained by simulating the image without overlapping cells and belt of inputs and with the same features added, are shown in Fig. $3 \mathrm{~b}$ and Fig. 3c, respectively. A clear lost of neighboring interaction is shown in Fig. 3b, which is recuperated by the overlapping and belt of inputs, shown in Fig. 3c.

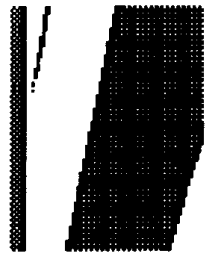

(a)

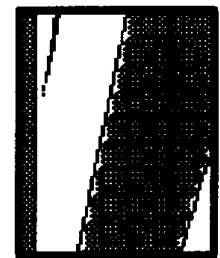

(b)

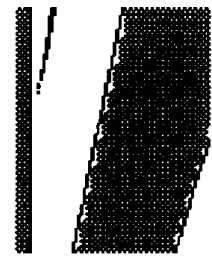

(c)
Fig. 3. Time-multiplexing simulation.

(a) Original image.

(b) Without overlapping \& belt of inputs.

(c) With overlapping of two \& belt of inputs.

One interesting aspect to be considered from time multiplexing simulations is the optimum block size of the CNN. Fig. 4 displays timing simulation results versus different block sizes. Solid line results correspond to computations that were carried out on a 125,235 pixel-image without black/white blocks and using an Averaging Template. In this case, it can be observed that beyond a block size of $50 \times 50$ no noticeable CPU time improvement is made. The results presented by the dashed line correspond to computations that were carried out using an Edge Detection template on an image with many black/white blocks. For smaller block sizes many black/white blocks were found which makes the computation efficient. Beyond block sizes of $80 \times 80$ no more black/white blocks were found. This is why this is the peak computation time. Fig. 5 shows the average number of iterations within each $\mathrm{CNN}$ block and for the whole image for the Averaging Template case. These results show the complexity of operations that must be considered when deciding the actual $\mathrm{CNN}$ hardware size.

\section{CONCLUSION}

While keeping the features of the raster simulator, the time-multiplexing simulator presented here process the image block by block, simulating $\mathrm{CNN}$ the way the hardware would, if the number of CNN processors of the hardware is smaller than the input image, which usually it is the case with practical size images.

With the overlapping and external belt of inputs, the neighboring interaction between $\mathrm{CNN}$ blocks is ensured, but at the same time, computation costs also increased. However, with the added feature of processing the all-black and all-white blocks just once for the entire simulation, the simulation time is brought down to the levels of raster simulation, if not better, in some cases, depending on the input image and the size of block and overlap chosen.

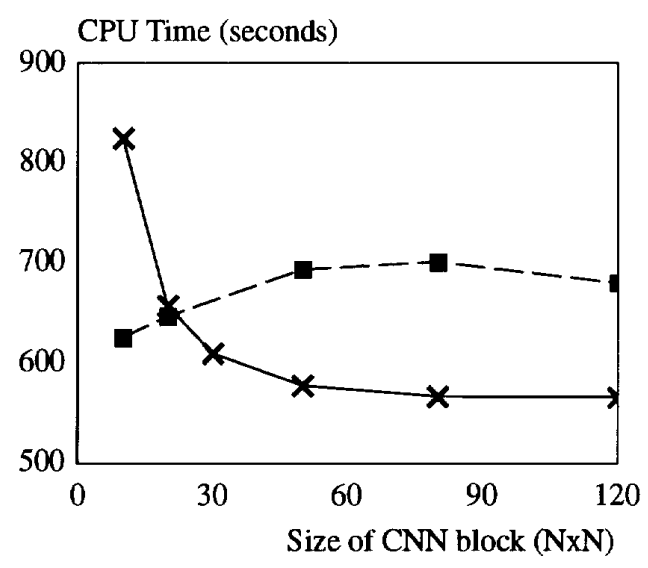

Fig. 4. CPU Time vs. CNN Block size

Number of iterations

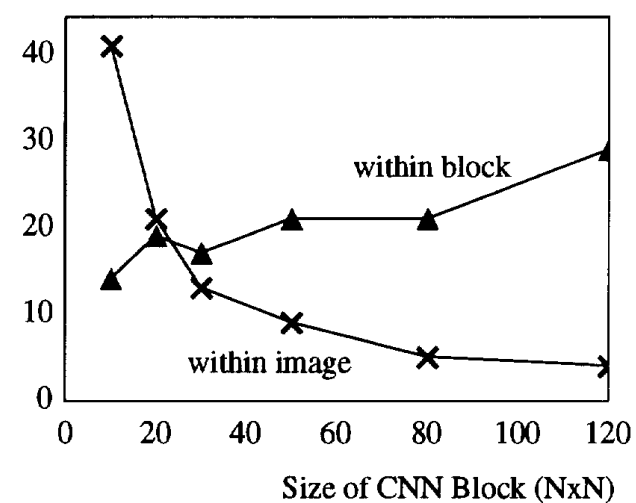

Fig. 5. Number of iterations vs. CNN Block Size

\section{REFERENCES}

[1] "Single-Layer Raster CNN Simulator", IEEE Int. Symposium on Circuits and Systems, London, 1994.

[2] L. O. Chua and L. Yang, "Cellular Neural Networks: Theory \& Applications," IEEE Trans. Circuits and Systems, Vol. CAS-35, pp. 1257-1290, 1988. 